\title{
An Observational Study of an Epilepsy Monitoring Unit in a Level 4 Epilepsy Center, Post-Implementation of the National Association of Epilepsy Centers 2010 Revised Guidelines
}

\author{
Mohankumar Kurukumbi ${ }^{\mathrm{a}, \mathrm{d}}$, Jose Castillo ${ }^{\mathrm{b}}$, Tulsi Shah ${ }^{\mathrm{b}}$, Joanne Lau $^{\mathrm{b}}$, Bhumi Patel ${ }^{\mathrm{b}}$, \\ Soumil Narayan ${ }^{\mathrm{b}}$, Laura Madarasz ${ }^{\mathrm{c}}$, Yun Fang ${ }^{\mathrm{c}}$, Caroline Shadowen ${ }^{\mathrm{b}}$, \\ Divya Sahajwalla ${ }^{b}$
}

\begin{abstract}
Background: Of the 2.7 - 3.4 million Americans estimated to have some form of epilepsy, approximately $25-30 \%$ of these individuals do not have adequate seizure control and suffer from intractable epilepsy. The objective of this study was to report outcomes of patients with epilepsy monitoring unit (EMU) admissions using data from a level 4 epilepsy center.
\end{abstract}

Methods: We performed a retrospective review of electronic medical records for 433 EMU patient visits between January 2016 and May 2019 at a level 4 comprehensive epilepsy center. The EMU protocols followed in these admissions were those listed in the guidelines by the National Association of Epilepsy Centers (NAEC). Patients were monitored by a medical team that included electroencephalogram technicians, neurophysiologists, and epileptologists.

Results: Of the 433 patients assessed, 384 met inclusion criteria. Mean length of stay in the EMU was 4 days. Of the patients, $73.4 \%$ had EMU stays resulting in new information which led to interventions including further diagnostic testing, surgical treatment, and medication changes. The most frequent intervention was a change in medication ( $68.8 \%$ of patients). Of the patients, $90.1 \%$ received a definitive diagnosis at the conclusion of their admission, with the most common diagnosis being epileptic seizures (66.7\%), followed by non-epileptic physiologic events $(14.3 \%)$ and psychogenic nonepileptic seizures $(8.6 \%)$.

Manuscript submitted August 4, 2021, accepted October 14, 2021

Published online October 26, 2021

${ }^{a}$ Inova Epilepsy Center, Inova Fairfax Medical Campus, Falls Church, VA, USA

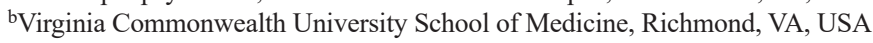
${ }^{\mathrm{C} N e u r o s c i e n c e}$ and Spine Institute Research, Inova Fairfax Medical Campus, Falls Church, VA, USA

${ }^{\mathrm{d}}$ Corresponding Author: Mohankumar Kurukumbi, Inova Epilepsy Center, Inova Fairfax Medical Campus, 3300 Gallows Rd., Falls Church, VA 22042,

USA.Email: mohan311@gmail.com

doi: https://doi.org/10.14740/jnr703
Conclusions: This study sought to describe outcomes from patients who stayed in our level 4 epilepsy center's EMU after the implementation of the revised NAEC guidelines made in 2010 . We investigated patient demographics as well as diagnosis and/or treatment changes after the EMU stay. We conclude that under the new NAEC guidelines, an EMU admission remains diagnostically useful in identifying if a patient has epilepsy or not. Our goal for this retrospective review is to inform future prospective outcomes studies and add to the body of literature demonstrating an EMU evaluation as a valuable diagnostic tool for epilepsy patients.

Keywords: Epilepsy; Seizure; Epilepsy monitoring unit; Admission; Level 4; NAEC guidelines

\section{Introduction}

Of the 2.7 - 3.4 million Americans estimated to have some form of epilepsy, over $30 \%$ of these individuals do not have adequate seizure control [1]. Most medical professionals would agree that patients with intractable epilepsy require evaluation at an epilepsy monitoring unit (EMU), which enables physicians to confirm a diagnosis of epilepsy or psychogenic nonepileptic seizures (PNES), characterize seizures, optimize antiepileptic drug (AED) regimens, and/or discuss possible need for advanced surgical management.

The National Association of Epilepsy Centers (NAEC) has largely defined the protocols of an EMU in a specialized epilepsy center, publishing their first guidelines in 1990. One study done in 2007 reviewed the diagnostic utility of an EMU post-implementation of the NAEC 2001 guidelines, reporting a diagnosis of epilepsy in $87.8 \%$ of admissions and a change in treatment in $79 \%$ of admissions [2]. Since then, an EMU for evaluation purposes has become a well-known medical tool, but there remains limited evidence about the implications of EMU admissions on patient outcomes.

The NAEC has published several more guideline revisions in the past two decades, with the most recent update published in 2010 [3]. Therefore, the objective of this study was to describe the outcomes of patients with EMU admissions at a 
level 4 epilepsy center after its implementation.

\section{Materials and Methods}

\section{Patient information/data points}

We performed a retrospective review of electronic medical records (EMRs) for 433 EMU patient visits between January 2016 and May 2019 at a level 4 comprehensive epilepsy center in Inova Fairfax Hospital (Fairfax, Virginia) [4]. Inclusion criteria included: patients over age 18 with an EMU admission and full hospital course. Exclusion criteria included: patients under 18 years of age, having an incomplete EMU hospital stay due to early discharge or absence of performed electroencephalogram (EEG). Demographic information, post-EMU diagnosis, and recommended intervention post-EMU course were also collected. Intracranial EEG evaluations were not included in this study.

All patients referred to our EMU had a clinical history of seizures. Whether a prior EMU stay or spot/routine EEG was done to confirm seizures was not collected in this study. The number of patients with poor compliance was collected by the epileptologist when screening patients for an EMU stay candidacy but for the purposes of this paper, this data point was not collected or reported.

Demographic information included age, gender, and race/ ethnicity. Gender options included either male and female and each patient was able to select how they self identified. Race/ ethnicity was broken down into five categories where each patient selected from the options of White, Black, Hispanic/Latino, Pacific Islander, Native American, Alaskan Native, Asian, or other. Marital status was also obtained. Hospital diagnoses were collected and included: no EMU events with no diagnosis, EMU events with no diagnosis, non-epileptic physiologic events, PNES, epileptic seizures, and psychogenic events and epilepsy. Information regarding the recommendations for intervention after the EMU stay included either a change in AED medication, implantation of a vagal nerve stimulator (VNS) or responsive neurostimulator (RNS), surgical resection of an epileptic lesion, phase II monitoring with placement of intracranial electrodes, an intracarotid sodium amobarbital procedure (Wada) to localize language and memory in the brain, or referral to other specialists.

Time from first seizure to EMU stay, length of stay at EMU for each patient, and complications associated with EMU stay and post-epilepsy surgery were also collected for this study.

The study is a retrospective chart review and reports data in an anonymized, aggregated manner and is therefore exempt from IRB approval. This study was conducted in compliance with the ethical standards of the responsible institution on human subjects as well as with the Helsinki Declaration.

\section{Data collection and analysis}

Study data were collected and managed using Research Electronic Data Capture (REDCap), a secure electronic data capture tool at Inova Fairfax Hospital. Statistical analyses were done using the software $\mathrm{R}$ after the data were imported from
REDCap. Descriptive statistics were used to analyze collected data. Frequency distribution tables were created to report quantitatively on the patient demographics, time of seizure onset to EMU stay, and outcomes after each EMU stay. Statistical significance was determined by the use of the Fisher exact test and Chi-square test to each collected data point pre-EMU stay with post-EMU stay outcomes in order to find a correlation. Only significant findings were presented in this paper.

\section{EMU protocol}

The EMU protocols followed were those listed in the 2010 guidelines created by the NAEC. Patients were admitted to a level 4 epilepsy facility at Inova Fairfax Hospital for a total of $3-5$ days. EEG electrodes were placed on patients using the $10-20$ electrode montage. A certified EEG technician was present to monitor the EEG in real time for epileptic activity and any other events during the EMU stay. Continuous video recording was performed throughout the study. The patient was also given a push button to press when they wanted to mark any events suggestive of seizurelike activity. The patients were subjected to sleep deprivation, photostimulation, and hyperventilation in attempts to provoke and capture seizure activity on video EEG (vEEG).

Safety features that were practiced included: keeping the head of the bed in the lowest position possible, recommending the use of "ear buds" if earphones were desired, instructing patients to call for their nurse if having difficulties with any of the wires/cables, showering only at the conclusion of their EMU stay, having bed rails that were padded, using a bed alarm that rang when patients exited their beds, and having a nurse available at all times if patients desired to step out of bed.

A board-certified neurophysiologist and epileptologist reviewed the study and made recommendations during and at the conclusion of the EMU stay. Diagnosis of an epileptic seizure was defined by clinical history of seizures and documentation of abnormal epileptiform discharges during their EMU monitoring as described by the International League Against Epilepsy (ILEA) in their 2017 positional paper classifying the epilepsies. Such epileptiform discharges included focal delta slowing and/or sharp waves, spike waves, or spike wave discharges. Additionally, events were also labeled seizures if the patient had a sudden change in behavior caused by electrical hypersynchronization of neuronal networks in the cerebral cortex.

Options for diagnosis consisted of: no EMU events with no diagnosis, EMU events with no diagnosis, non-epileptic events (did not meet the criteria for epileptic or psychogenic events), PNES (patient documented behavioral seizure-like events with no epileptiform correlation on EEG), epileptic seizures, and psychogenic events and epilepsy. The types of epilepsy discovered were classified as either focal or generalized. For those that had focal types of epilepsy, only the category of having focal epilepsy was collected and the type of intervention they underwent. Patients were not screened for sudden unexpected death in epilepsy (SUDEP) prior to their EMU stay.

All patients underwent a follow appointment with the same epileptologist who recommended and oversaw every patient's EMU stay. Focus positive patients underwent a neurosurgical consultation with a board certified functional neurosurgeon, 


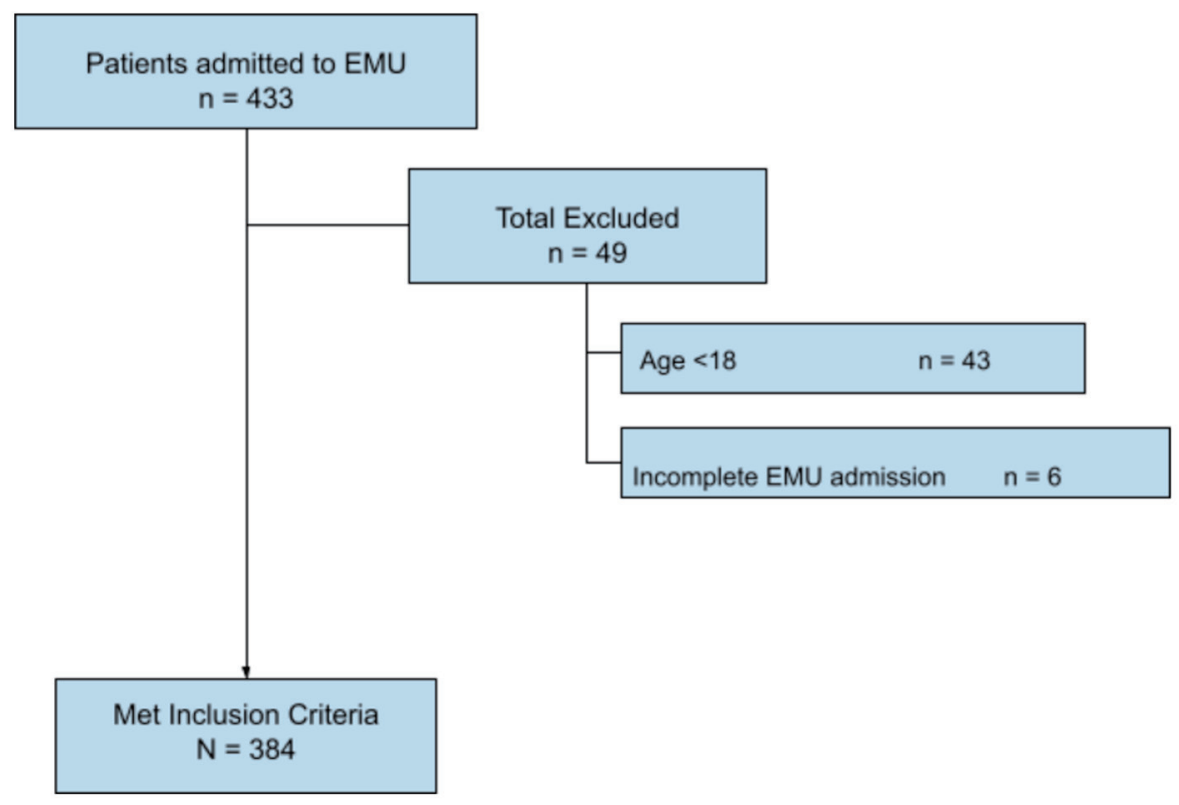

Figure 1. Flow diagram of patient inclusion and exclusion. EMU: epilepsy monitoring unit.

to determine if further diagnostic testing would be fruitful, or if resection surgery could be of benefit. Seizure control postsurgery was not recorded for the purposes of this study.

\section{Results}

\section{Patient population}

Of the 433 patients that were assessed, a total of 384 patients met inclusion criteria and were included in the final analysis. Forty-nine patients were excluded due to age and incomplete EMU admission (Fig. 1). No patients had lesional magnetic resonance imaging (MRI) prior to their EMU stay.

\section{Demographics}

The majority of patients were female $(60.9 \%)$ and the average age was 40.8 years old (standard deviation: \pm 17 ). White/ Caucasians comprised the majority of the patients analyzed (55.9\%), with African Americans being the second most common (19.3\%), followed by Latino, Asians, American Indians, and Native Pacific Islanders. A notable number of patients (10.2\%) either chose not to list their ethnicity or identified as "other," which was not further described. Patient demographics are listed in Table 1.

Table 1. Demographics of Age, Gender, and Ethnicity Stratified in Groups That Received Intervention Versus Those Having No Change in Management

\begin{tabular}{llll}
\hline & Total & Intervention & No change \\
\hline Age & $40.8 \pm 17$ & $40.2 \pm 16.9$ & $42.5 \pm 17.4$ \\
Gender & & & 37 \\
$\quad$ Male & 150 & 113 & 65 \\
$\quad$ Female & 234 & 169 & 59 \\
Ethnicity & & 156 & 13 \\
$\quad$ White & 215 & 61 & 7 \\
$\quad$ Black/African American/African & 74 & 21 & 10 \\
$\quad$ Latino/Hispanic & 28 & 13 & 1 \\
$\quad$ Asian/South Asian & 23 & 2 & 1 \\
American Indian or Native Alaskan & 3 & 1 & 11 \\
Native Hawaiian/other Pacific Islander & 2 & 28 & \\
Other/not reported & 39 & & \\
\hline
\end{tabular}


Table 2. Diagnostic Outcomes of Patients With EMU Admissions

\begin{tabular}{|cl|}
\hline & Frequency (\%) \\
\hline Diagnosis received & $346(90.1 \%)$ \\
Epileptic seizures & $256(66.7 \%)$ \\
Non-epileptic events & $55(14.3 \%)$ \\
Psychogenic non-epileptic seizures & $33(8.6 \%)$ \\
Psychogenic events and epilepsy & $2(0.5 \%)$ \\
No diagnosis received & $38(9.9 \%)$ \\
EMU events, no diagnosis & $5(1.3 \%)$ \\
No events, no diagnosis & $33(8.6 \%)$ \\
\hline
\end{tabular}

EMU: epilepsy monitoring unit.

\section{Diagnosis}

Of the total 384 patients admitted for an EMU stay, 90.1\% received a definitive diagnosis at the conclusion of admission. Epileptic seizures were diagnosed in the majority of patients $(66.7 \%)$. Non-epileptic events were the second most common definitive diagnosis (14.3\%), followed by PNES (8.6\%). Two patients were found to have both PNES and epileptic seizures. Only $8.6 \%$ of patients had no events during their EMU stay and resulted in no diagnosis, while $1.3 \%$ experienced equivocal events resulting in no diagnosis (EMU events with no diagnosis). Neither of these last two groups received definitive diagnosis at the end of their hospital course. Table 2 shows more extensive review of outcomes. Of the group diagnosed with epilepsy, more than $50 \%$ had generalized seizures. No complications were noted post-EMU stay and post-epilepsy surgery.

\section{Intervention}

The mean length of stay at the EMU was 4 days (range 3 - 5).
EMU stays for $73.4 \%$ of patients resulted in new information, leading to a change in management of their disease. Interventions that were implemented included further diagnostic testing, surgical resection, VNS, RNS, and medication changes (Table 3). All patients undergoing RNS also underwent diagnostic intervention such as phase II monitoring or Wada. The most common intervention recommended $(68.8 \%)$ was a change in medication, which included the removal and/or addition of $A E D(s)$. VNS was the most common surgical intervention recommended as a result of the EMU stay. Long-term outcomes are currently being collected and reviewed by our research team since the creation of this paper.

\section{Discussion}

Epilepsy is defined as a chronic state of the brain that makes it susceptible to the generation of seizures [5]. In the United States of America, epilepsy causes a significant burden on the population, with over 3 million adults dealing with the disease in $2015[6,7]$. In the state of Virginia alone, there are an estimated 85,000 cases of epilepsy [8], with almost a third of that number concentrated in the Northern Virginia area, where our epilepsy center is located [9].

With this study, we aimed to describe outcomes from a level 4 epilepsy center post-implementation of the updated NAEC guidelines published in 2010. Prior to the new guidelines published in 2010, one study done by Smolowitz et al in 2007 showed that over $87 \%$ of admissions to an EMU resulted in a definitive diagnosis [2]. This is remarkably similar to our study, in which $91 \%$ of participants received a definitive diagnosis at the conclusion of their EMU stay. We conclude that with the new guideline implementation, an EMU admission remains diagnostically useful in identifying if a patient has epilepsy or not.

At our institution, the mean length of stay in the EMU was 4 days, with a range of 3 - 5 days. The literature regarding a recommended length of stay varies among EMUs across

Table 3. Interventions as a Result of EMU Evaluation

\begin{tabular}{|ll}
\hline & Frequency (\%) \\
\hline Intervention performed & $282(73.40 \%)$ \\
Medication & $264(68.80 \%)$ \\
Medication change & $264(68.80 \%)$ \\
Surgical intervention & $53(13.80 \%)$ \\
Resection & $2(0.52 \%)$ \\
RNS & $6(1.56 \%)$ \\
VNS & $45(11.70 \%)$ \\
Intervention by diagnostic studies & $23(5.99 \%)$ \\
Wada & $9(2.34 \%)$ \\
Intracranial electrode placement/phase II monitoring & $14(3.65 \%)$ \\
No intervention performed & $102(26.60 \%)$ \\
\hline
\end{tabular}

Interventions not mutually exclusive and total frequency of interventions may exceed the number of enrolled patients. EMU: epilepsy monitoring unit; RNS: responsive neurostimulator; VNS: vagus nerve stimulator; Wada: intracarotid sodium amobarbital procedure. 
the United States of America, with no real "rule of thumb", and sometimes with epileptologists' bias playing a role. One paper showed that the length of stay varied based on the clinical question that the EMU stay was attempting to answer, for example, patients admitted for presurgical evaluation stayed longer compared to those admitted for seizure characterization [10]. A similar study found that patients admitted to an EMU for the purposes of presurgical evaluation were typically kept on the unit longer (mean 7.1 days) versus patients admitted for event characterization or medication adjustment [11]. In our study, the main indication for an EMU stay was AED optimization $(43.8 \%)$.

Both the 2001 and updated 2010 NAEC guidelines do not offer specific guidance on length of stay adequacy, ultimately leaving it up to each epilepsy center's discretion. What the guidelines do standardize is the length of a vEEG, stating that this should be at least $24 \mathrm{~h}$ long. This is consistent with studies performed in a critical care unit, which demonstrated that $80-95 \%$ of patients with non-convulsive seizures will have an identifying event within $24-48 \mathrm{~h}$ of admission [12], and beyond 3 days of monitoring, the probability of new seizure activity decreases dramatically [13]. This evidence, along with the risk of adverse events associated with prolonged hospital stay and excess cost to patient and institution [14], guided our facility in determining EMU length of stay. This information demonstrates and adds to the fact that length of stay is still an important factor that needs to be further investigated.

We feel the unit length of stay should depend on many factors, some medical (e.g., the purpose of the evaluation, medical comorbidities that put patients at increased risk of adverse events), and some social (e.g., how long patients can be off work or away from household duties). Clinicians should consider all of these factors and use shared decision-making when discussing EMU admission with patients. The NAEC should consider these factors when creating future recommendations. There is no "one size fits all" for how long a patient needs to be admitted to the EMU for their stay to be considered adequate, but our findings suggest that a shorter stay can still yield critical diagnostic information.

With our paper, we also intended to bring some more insight into how patient demographics are associated with an EMU stay. Though epilepsy is experienced universally, it has been shown that the impact of epilepsy varies by sex, race, and socioeconomic status $[15,16]$. A scoping review of epilepsyrelated deaths in the United States of America from 2005 to 2014 revealed that Black/African-American patients had a death rate from the disease of 1.42 out of 100,000, compared to 0.86 per 100,000 for Caucasians and 0.70 per 100,000 for Latinx patients [17]. Black/African-American people with epilepsy are consistently undertreated in the categories of length of time to formal diagnosis, medication management, and the offering of surgical intervention for refractory epilepsy [18]. We advocate for further studies to investigate this question and hopefully improve quality of care for all patients suffering from epilepsy, especially for Black/African-American patients, who have historically been undertreated.

Finally, to touch on the surgical outcomes of our patients, we found that most of the patients receiving surgical intervention $(11.7 \%)$ had a VNS placed. In review of the literature,
Smolowitz et al in 2007 reported that $18.63 \%$ of their patients underwent surgery within 3 years. Unfortunately, they did not elaborate on which procedures were performed. Another article showed that roughly $23 \%$ of patients went on to have surgery referrals, with only $18 \%$ having surgeries [19].

We hypothesize that differences in surgical interventions in our study versus what is seen in the literature could be due to the type of seizure discovered in our group, and, our population demographics. VNS was a largely preferred method of treatment in our group, possibly secondary to the fact that most of our patients had generalized seizures and the fact that VNS is a less invasive procedure, making it more appealing to our population based on subjective interpretation by our epilepsy team who screened all of our EMU candidates.

\section{Limitations}

Because of the retrospective nature of this study, the data were collected not for primary research intentions but for patient care. Therefore, the conclusions able to be drawn are limited. However, our institution does receive numerous referrals from around the region and therefore serves a very diverse patient population, increasing demographic diversity of our study. Our goal is for this study to improve patient compliance, as well as inform further research regarding the utility of EMU in the diagnosis and management of epilepsy. We did not report the disease trajectories of those patients left undiagnosed in our study, as the goal of our study was to report on the diagnosis and treatment of epilepsy patients, not non-epileptic patients.

\section{Conclusions}

This study sought to describe outcomes from patients who stayed in our level 4 epilepsy center's EMU after the implementation of the revised NAEC guidelines made in 2010 . We investigated patient demographics as well as diagnosis and/or treatment changes after the EMU stay. We conclude that under the new NAEC guidelines, an EMU admission remains diagnostically useful in identifying if a patient has epilepsy or not. Interestingly we also saw that the breakdown in the diagnostic category did differ between the 2001 and 2010 NAEC guidelines which we postulate to be secondary to the EMU length of stay and patient population, when compared to findings from the current published literature. Our goal for this retrospective review is to inform future prospective outcomes studies and add to the body of literature demonstrating an EMU evaluation as a valuable tool in improving the quality of care for epilepsy patients.

\section{Acknowledgments}

The authors would like to thank all of the nurses, and EEG technologists of the Epilepsy Monitoring Unit at Inova Fairfax Hospital under the leadership of Becky Schrews RN (Epilepsy Navigator), Mike Schmiester (Manager Neurodiagnostics), 
Holly Wirth (Director In-patient Unit) and Anne M. Giles (Nurse Practitioner).

\section{Financial Disclosure}

This research did not receive any specific grant from funding agencies in the public, commercial, or not-for-profit sectors.

\section{Conflict of Interest}

The authors report no conflict of interest.

\section{Informed Consent}

The study is a retrospective chart review and reports data in an anonymized, aggregated manner with no possibility of linking specific patients to data reported and is therefore exempt from requiring informed consent of participants.

\section{Author Contributions}

All authors certify that he or she has participated sufficiently in the intellectual content, the analysis of data. Each author has reviewed the final version of the manuscript and approves it for publication. Mohankumar Kurukumbi was the principal investigator and acted as a supervisor in all aspects of data collection and analysis, as well as a key part of manuscript writing. Jose Castillo performed data collection, analysis, and manuscript writing. Tulsi Shah performed data collection, analysis, and manuscript writing. Joanne Lau performed data collection and analysis. Bhumi Patel performed data collection and analysis. Soumil Narayan performed data collection and analysis. Laura Madarasz performed data collection and analysis. Yun Fang performed data collection and analysis. Caroline Shadowen was a key part of the manuscript writing, editing, and submission. Divya Sahajwalla was a key part of manuscript writing.

\section{Data Availability}

The authors declare that data supporting the findings of this study are available within the article.

\section{Abbreviations}

AED: antiepileptic drug; CDC: Centers for Disease Control and Prevention; CT: computed tomography; EEG: electroencephalogram; EMU: epilepsy monitoring unit; ILAE: International League Against Epilepsy; LMIC: low and middleincome countries; MRI: magnetic resonance imaging; NAEC: National Association of Epilepsy Centers; PNES: psychogenic non-epileptic seizures; REDCap: Research Electronic Data
Capture; RNS: responsive neurostimulator; SUDEP: sudden unexpected death in epilepsy; vEEG: video electroencephalogram; VNS: vagus nerve stimulator; Wada: intracarotid sodium amobarbital procedure

\section{References}

1. Kwan P, Brodie MJ. Early identification of refractory epilepsy. N Engl J Med. 2000;342(5):314-319.

2. Smolowitz JL, Hopkins SC, Perrine T, Eck KE, Hirsch LJ, O'Neil Mundinger M. Diagnostic utility of an epilepsy monitoring unit. Am J Med Qual. 2007;22(2):117-122.

3. Labiner DM, Bagic AI, Herman ST, Fountain NB, Walczak TS, Gumnit RJ, National Association of Epilepsy C. Essential services, personnel, and facilities in specialized epilepsy centers-revised 2010 guidelines. Epilepsia. 2010;51(11):2322-2333.

4. Inova Epilepsy Center. Inova. https://www.inova.org/ our-services/inova-epilepsy-center. Accessed August 18, 2020.

5. Beghi E. The epidemiology of epilepsy. Neuroepidemiology. 2020;54(2):185-191.

6. Epilepsy. World Health Organization. https://www.who. int/news-room/fact-sheets/detail/epilepsy. Published June 20, 2019. Accessed August 5, 2020.

7. Epilepsy Data and Statistics. Centers for Disease Control and Prevention. https://www.cdc.gov/epilepsy/data/index.html. Published January 25, 2019. Accessed August 5, 2020.

8. Zack MM, Kobau R. National and state estimates of the numbers of adults and children with active epilepsy United States, 2015. MMWR Morb Mortal Wkly Rep. 2017;66(31):821-825.

9. Shafer PO, Sirven JI. Facts about seizures and epilepsy. Epilepsy Foundation. https:/www.epilepsy.com/learn/ about-epilepsy-basics/facts-about-seizures-and-epilepsy. Published July 2013. Accessed August 24, 2020.

10. Lampe E, Forster J, Herbst E, Spitz M, Frey L. Pre-admission clinical factors affect length of stay in the epilepsy monitoring unit. Neurodiagn J. 2014;54(2):138-147.

11. Gazzola DM, Thawani S, Agbe-Davies O, Carlson C. Epilepsy monitoring unit length of stay. Epilepsy Behav. 2016;58:102-105.

12. Caricato A, Melchionda I, Antonelli M. Continuous electroencephalography monitoring in adults in the intensive care unit. Crit Care. 2018;22(1):75.

13. Westover MB, Shafi MM, Bianchi MT, Moura LM, O'Rourke D, Rosenthal ES, Chu CJ, et al. The probability of seizures during EEG monitoring in critically ill adults. Clin Neurophysiol. 2015;126(3):463-471.

14. Mitchell RJ, Herkes G, Nikpour A, Bleasel A, Shih P, Vagholkar S, Rapport F. Examining health service utilization, hospital treatment cost, and mortality of individuals with epilepsy and status epilepticus in New South Wales, Australia 2012-2016. Epilepsy Behav. 2018;79:9-16.

15. Ali A. Global health: epilepsy. Semin Neurol. 2018;38(2):191-199.

16. Bangar S, Shastri A, El-Sayeh H, Cavanna AE. Women 
with epilepsy: clinically relevant issues. Funct Neurol. 2016;31(3):127-134.

17. Greenlund SF, Croft JB, Kobau R. Epilepsy by the Numbers: Epilepsy deaths by age, race/ethnicity, and gender in the United States significantly increased from 2005 to 2014. Epilepsy Behav. 2017;69:28-30.

18. Margolis SA, Gonzalez JS, Spindell J, Mohamadpour M,
Grant AC, Nakhutina L. Assessment of medication management capacity in a predominantly African American and Caribbean American sample of adults with intractable epilepsy. Epilepsy Behav. 2018;88:308-314.

19. Benbadis SR, O'Neill E, Tatum WO, Heriaud L. Outcome of prolonged video-EEG monitoring at a typical referral epilepsy center. Epilepsia. 2004;45(9):1150-1153. 